\title{
Communications
}

\section{Formation of CdS Nanoparticles within Cubic Mesoporous Silica Thin Films}

\author{
Kashinath Rangu Patil, Young Kyu Hwang, Deok-Kyu Kim, Jong-San Chang, and Sang-Eon Park ${ }^{\dagger, *}$ \\ Research Center for Nanocatalysts, Korea Research Institute of Chemical Technology, P.O. Box 107, Yuseong, \\ Daejeon 305-306, Korea. "E-mail: jschang@pado.krict.re.kr \\ †Department of Chemistry, Inha University, Incheon 402-751, Korea \\ Received October 6, 2004
}

Key Words : Mesoporous thin films, CdS, Nanoparticles, Cubic

The self assembly of nanoparticles into ordered morphologies is one of the topics in focus field of materials science as it is expected to create nanostructured materials with unique physical and chemical properties. ${ }^{1}$ Mesoporous silica materials possessing well ordered and controllable mesopores with a narrow pore size distribution have stimulated great interest, owing to their potential applications in catalysis, adsorption and nanotechnology. ${ }^{2}$ Despite the excellent control of pore size, early mesoporous materials were made in the form of powders, precluding their use in thin film applications like membranes, low dielectric constant interlayers, and optical sensors. ${ }^{3}$ For transport precursor molecule or ions for assembly of nanoparticles or nanorods inside the channels of mesoporous oxides three methodologies have been developed. They are (i) the direct impregnation of mesoporous materials with precursor molecules or ions, ${ }^{4}$ (ii) use of functional ligands to randomly fictionalize internal surface of mesoporous oxides, followed by loading of precursor compounds through strong interaction between the functional ligands and metal ions ${ }^{5}$ and (iii) ion exchange reaction. ${ }^{6}$

Several semiconductor compounds, e.g. $\mathrm{CdS},{ }^{7} \mathrm{CdSe},{ }^{8}$ $\mathrm{GaAs}^{9}$ have been implanted to study the growth and the properties of quantum dots confined in an inorganic porous matrix. $\mathrm{CdS}$ is a very attractive semiconductor material due to its stability, photochemical properties and ease of preparation and handling as well as the practical applications in photonics, electronics etc. ${ }^{10}$

It is reported in the literature that $\mathrm{CdS}$ nanoparticles have been formed inside the ordered mesoporous materials via an ion-exchange reaction followed by sulfurization with sulphur precursor solution. ${ }^{6}$ Also $\mathrm{CdS}$ nanoparticles are reported to be formed inside modified mesoporous aluminosilicate MCM-41 and siliceous SBA-15 through a reaction with the silylation reagent which served to preposition the sulfide source within the mesoporous channels. ${ }^{11}$ Recently, CdS nanoparticles were incorporated into the mesoporous hexagonal SBA-15 thin films by electron depositions route. ${ }^{12}$ However, mesoporous thin films with three-dimensional cubic structures are of particular interest for encapsulation of semiconductor because their pores are more easily accessi- ble than those of other anisotropic structures.

In this regards, we focus on the preparation of $\mathrm{CdS}$ nanoparticles into the pores of mesoporous cubic silica film by an approach of impregnation followed by post treatment.

A thin film of mesoporous materials (SBA-16) was coated on silicon and glass substrate using an Evaporation Induces Self Assembly (EISA) following reported procedure. ${ }^{13}$ Final molar ratio is TEOS : $\mathrm{EtOH}: \mathrm{H}_{2} \mathrm{O}: \mathrm{HCl}: \mathrm{F} 127: \mathrm{PPO}=1$ : $20: 5: 0.004: 0.0046: 0.0023$. The loading of precursors leading to $\mathrm{CdS}$ nanoparticles was carried out by the sequential incipient wetness method, and initially the calcined film was immersed in $\mathrm{Cd}$ acetate $0.01 \mathrm{wt} . \%$ in ethanol under reduced pressure. The surface was washed with ethanol immediately after the film was drawn-out from the solution with ethanol and dried under vacuum. The metal loaded film was treated with $\mathrm{H}_{2} \mathrm{~S}$ in ethanol. Subsequently, the film was washed with ethanol and characterized by EDX, XRD, BET, TEM and UV-visible spectroscopy. The formation of CdS was tested by subjecting the film to EDX and XP analysis. The atomic concentration ratio of $\mathrm{Cd}$ and $\mathrm{S}$ was almost equal in EDX analysis. XP measurements have been carried out on CdS encapsulated in SBA-16 to confirm the formation of $\mathrm{CdS}$ material within SBA-16 even after washing. The wide scan spectra showed the presence of $\mathrm{Cd}$ and $\mathrm{S}$ along with $\mathrm{Si}$ and $\mathrm{O}$ from SBA-16. The binding energies of $\mathrm{Cd} 3 \mathrm{~d}_{5 / 2}, \mathrm{Cd} 3 \mathrm{~d}_{3 / 2}$ and $\mathrm{S} 2 \mathrm{p}$ of the sample are 405.0, 411.6 and $161.5 \mathrm{eV}$, respectively, which are in good agreement with the literature values. ${ }^{14}$ The quantification of the peak gives the ratio of $1.0: 0.95$ for $\mathrm{Cd}$ to $\mathrm{S}$. It is reasonable, therefore, to infer the formation of stoichiometric amounts of CdS by the given reaction.

Low angle XRD patterns of calcined SBA-16 and CdS particles within SBA-16 are shown in Figure 1. Two peaks are indexed corresponding to (110), (211) a cubic unit cell for SBA-16. ${ }^{13}$ The XRD patterns of SBA 16 and CdSSBA16 are very similar. Some small angle changes can be noted as follows: CdS-SBA-16 peaks are broader as compared to the peak observed for SBA-16 and CdS-SBA16 peaks are slightly shifted to higher value of $2 \theta$ scale which may be a result from partial shrinkage of the mesoporous film by further condensation of the silicate in the incorpo- 


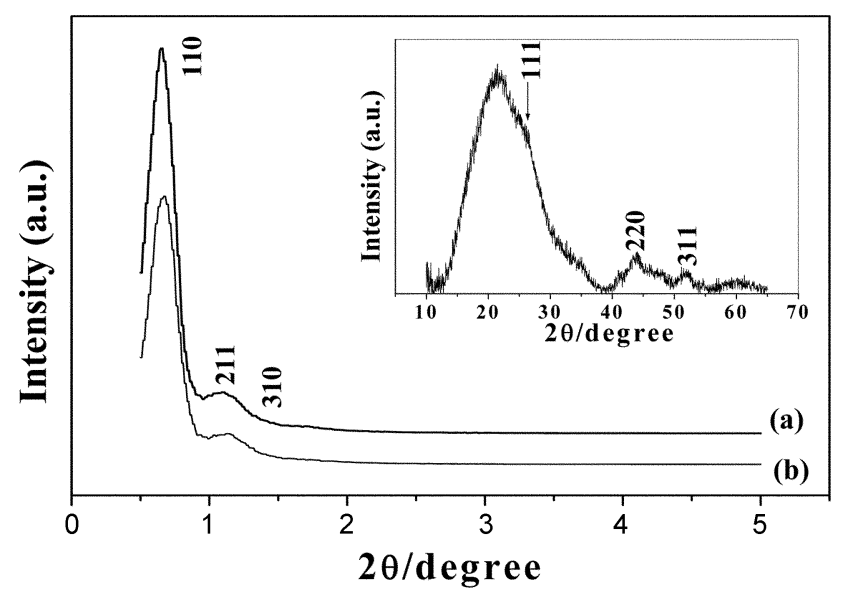

Figure 1. XRD patterns of calcined mesoporus thin films: (a) pure silica films and (b) after CdS inocorporated. Inset is wide-angle XRD pattern of CdS in silica film.

ration process. ${ }^{15}$ The intensity of the peaks are also decreased. The inset of Figure 1 shows the wide-angle XRD pattern for CdS-SBA-16 that contains cadmium sulfide nanoparticles. The three broadened peaks are matching to the peaks assignable to (111), (220) and (311) lattice planes of cubic crystalline CdS, respectively. The presence of a broad peak at a $2 \theta$ value of about $21^{\circ}$ is attributed to the amorphous structure of silica. The average particle size estimated from the Debye-Scherrer formula is about 3 to 4 $\mathrm{nm}$, which is smaller than the excitonic length of CdS, $6 \mathrm{~nm}$. The band gap was widened to $2.6 \mathrm{eV}$. The SBA- 16 thin films are very stable during the $\mathrm{CdS}$ encapsulation.

The $\mathrm{N}_{2}$ adsorption-desorption isotherm of the preformed silica host and CdS loaded mesoporous silica sample shows the type IV behavior with a hysteresis loop (not shown). The specific surface area evaluated by BET equation decreased 340 to $282 \mathrm{~m}^{2} / \mathrm{g}$ for the CdS loaded sample, implying the occupation of $\mathrm{CdS}$ particles within mesopores. This considerable decrease in surface area supports the formation of CdS inside the pores.

The TEM image of CdS-SBA-16 is shown in Figure 2, where the uniformly placed SBA-16 with dispersed CdS particles are observed. The cubic mesopores of the host material SBA-16 were unaffected by the presence of CdS inside the pores, indicating that the present technique does not cause significant deterioration of the SBA-16 framework. The $\mathrm{d}_{110}$ value calculated from TEM image is in agreement with the value from XRD.

UV-visible spectra of CdS-SBA-16 shows an absorption onset at $470 \mathrm{~nm}$. Bulk CdS show absorption onset at $510 \mathrm{~nm}$. The blue shift from 510 to $470 \mathrm{~nm}$ of the absorption edge can be attributable to of size quantization.

In conclusion, the nanocomposites consisting of $\mathrm{CdS}$ within the pores of cubic mesoporous films were synthesized by the incipient wetness method. Characterization results of EDX, XPS, XRD, BET, TEM and optical absorption proved that $\mathrm{CdS}$ nanoparticles are confined in the

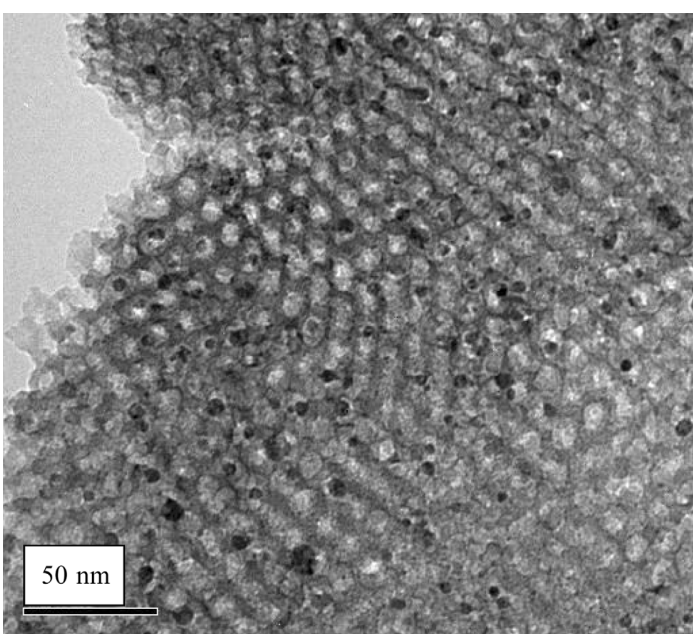

Figure 2. TEM image of CdS nanoparticles encapsulated in mesoporous silica thin film.

pores of mesoporous cubic silica (SBA-16). Thin film remains intact after the incorporation of $\mathrm{CdS}$ nanoparticles.

This work was supported by the Korea Ministry of Commerce, Industry and Energy (MOCIE) through the Research Center for Nanocatalysis and Institutional Research Program (KRICT). The authors thank W. Lee and Prof. Y. -U. Kwon for the low-angle XRD measurement. K. R. Patil greatfully acknowledge the financial support by KOFST.

\section{References}

1. Braun, P. U.; Osenar, P.; Stupp, S. Nature 1996, 380, 325.

2. (a) Kresge, C. T.; Leonowicz, M. F.; Roth, W. J.; Vartuli, J. C.; Beck, J. L. Nature 1992, 359, 710. (b) Park, D. H.; Park, S. S.; Choe, S. J. Bull. Kor. Chem. Soc. 1999, 20, 291.

3. (a) Lu, Y.; Ganguli, R.; Drewien, C.; Anderson, M.; Brinker, C. J.; Gong, W.; Guo, Y.; Soyez, H.; Dunn, B.; Huang, M.; Zink, J. Nature 1997, 389, 364. (b) Wu, C.; Han, G.; Bein, T. Science 1994, $264,1757$.

4. Han, Y. J.; Kim, J. M.; Stucky, G. D. Chem. Mater. 2000, 12, 2068

5. Zang, W. H.; Shi, J. L.; Wang, L. Z.; Yan, D. S. Chem. Mater. 2000, 12, 1408

6. Zang, Z.; Dai, S.; Fan, X.; Blom, D.; Pennycook, S. J.; Wei, Y. J. Phys. Chem. B 2001, 105, 6755.

7. (a) Wang, S.; Choi, D.-G.; Yang, S.-M. Adv. Mater. 2002, 14, 1311. (b) Choi, S. W.; Yoon, J.-H.; An, M. J.; Chae, W. S.; Cho, H. M.; Choi, M.-G.; Kim, Y.-R. Bull. Korean Chem. Soc. 2004, 25, 983.

8. Parala, H.; Winkler, H.; Kolbe, M.; Wohlfart, A.; Fischer, R. A.; Schmechel, R.; Seggern, H. Adv. Mater. 2000, 12, 1050.

9. Srdanov, V. I.; Alxneit, I.; Stucky, G. D.; Reaves, C. M.; DenBaars, S. P. J. Phys. Chem. B 1998, 102, 3341.

10. Lowndes, D. H.; Fowlkes, J. D.; Pedraza, A. J. Appl. Surf. Sci. 2000, 154, 647.

11. Xu, W.;Liao, Y.; Akins, D. L. J. Phys. Chem. B 2002, 106, 11127.

12. Gu, J.; Shi, J.; Chen, H.; Xiong, L.; Shen, W.; Ruan, M. Chem. Lett. 2004, 33, 828.

13. Hwang, Y. K.; Patil, K. R.; Park, S.-E.; Chang, J.-S. Micropor. Mesopor., Mater. 2005, 78, 245.

14. Nanda, J.; Kuruvilla, B. A.; Sharma, D. D. Phy. Rev. B 1999, 59 , 7473.

15. Fukuoka, A.; Araki, H.; Sakamoto, Y.; Sugimoto, N.; Tsukada, H.; Kumai, Y.; Akimoto, Y.; Ichikawa, M. Nano Letters 2002, 2, 793. 